\title{
Decompression of the labyrinthine segment of the facial nerve in acoustic neuroma surgery: a consideration for minimizing postoperative delayed facial nerve dysfunction
}

\section{Michael J. Holliday, M.D., and Prakash Sampath, M.D.}

Departments of Otolaryngology-Head and Neck Surgery and Neurological Surgery, The Johns Hopkins Hospital, Baltimore, Maryland

Delayed facial nerve palsy, a condition characterized by spontaneous deterioration of facial nerve function in patients who had otherwise normal or near-normal facial function in the immediate postoperative period, has been reported in 15 to $29 \%$ of patients undergoing microsurgical resection of vestibular schwannomas. One putative mechanism for its occurrence suggests that edematous entrapment of the facial nerve in the meatal foramen (the narrowest segment of the internal auditory canal) may lead to nerve ischemia or necrosis and subsequent facial nerve dysfunction. To assess whether meatal decompression may help reduce the incidence of delayed facial nerve palsy during microsurgical resection of acoustic tumors, we compared 25 patients undergoing translabyrinthine removal of acoustic neuromas who received prophylactic decompression of the labyrinthine segment of the facial nerve (Group 1) with 40 patients who did not receive facial nerve decompression (Group 2). No patients in Group 1 had a delayed progressive facial paralysis with degeneration. In contrast, when Group 2 patients with larger, average-sized tumors were reviewed, eight patients $(20 \%)$ developed delayed degeneration. These findings suggest that decompression of the labyrinthine segment may be of value in acoustic tumor surgery in reducing delayed facial nerve dysfunction. Further study is indicated in this important area.

Key Words * acoustic neuroma * facial nerve * delayed palsy * decompression * labyrinthine

Since the onset of microsurgical procedures for acoustic neuromas, remarkable progress has been made in the anatomical preservation of the facial nerve.[18] In recent years, many series of patients with acoustic neuromas have been reported that show good maintenance of seventh cranial nerve function. $[2,4,12,14,16,17]$ The question of ultimate facial nerve function, however, is often quite vague because terms such as good, fair, and normal are being used with meanings that differ from surgeon to surgeon. Brackmann and Barrs[3] were the among the first to stress the importance of developing a standard grading system for comparison of results following acoustic neuroma surgery; subsequently, House and Brackmann[10] proposed a six-point scale for grading facial nerve recovery that was adopted by the Facial Nerve Disorders Committee of the American Academy of Otolaryngology-Head and Neck Surgery as the universal standard and is now widely used. Despite great advances in our understanding of 
intra- and postoperative mechanisms of facial nerve injury, there are still many unresolved questions in facial nerve preservation after surgery for acoustic neuromas.

One poorly understood phenomenon is the development of postoperative delayed facial nerve dysfunction. $[8,11,20]$ This condition is characterized by initial excellent postoperative facial expression that deteriorates into complete paralysis and degeneration of the nerve with ultimate regenerative synkinetic action.[13] Many mechanisms have been postulated as the cause of delayed facial nerve palsy $[8,12]$ and include edema within the perineurium of the nerve, vasospasm, venous engorgement, and immunological reactions, as well as traction on the nerve from cerebrospinal fluid leak or brain swelling. Another theoretical mechanism is compression of the facial nerve in the labyrinthine segment of the temporal bone.[1,6] Consequently, some authors have advocated meatal decompression of the facial nerve during translabyrinthine acoustic neuroma approaches. $[9,19]$ Considerable investigation into neurotmesis and its avoidance has been accomplished in idiopathic facial palsy, herpes zoster oticus, and traumatic facial palsy.[6] In anatomical studies of temporal bones Dobozi[5] has demonstrated that the fallopian canal is narrowest in relation to facial nerve diameter in its labyrinthine segment. Gantz, et al.,[7] have shown with intraoperative evoked electromyography (EMG) in idiopathic palsies that the block to axonal impulse conductivity is found in the labyrinthine segment.

The value of decompression of the labyrinthine segment of the facial nerve in impending degeneration, however, is not altogether clear, because it is accepted as effective by some authorities and debated by others.[1] In contrast, the natural history of acute idiopathic facial palsy has been well studied by Peiterson,[15] who has demonstrated that if a palsy does not progress to complete axonal neurotmesis, beginning motion is seen in all cases within 4 weeks. However, when total nerve degeneration occurs, there is no observable evidence of voluntary facial function prior to 12 weeks after onset. We know that with nerve injury in the parotid distal facial branch area, we may see return of function beginning earlier than 12 weeks despite the occurrence of nerve degeneration. This correlates well with the distance through which regeneration must occur. Axoplasmic regeneration begins on Ravnier's node proximal to the injury and proceeds distally at the rate of approximately $1 \mathrm{~mm} /$ day, with individual moderate variation. If we presume that in acoustic neuroma surgery, the injury in a degenerated facial nerve may occur anywhere from the brainstem root entry zone (exit point) to the second genu near the lateral semicircular canal, we would expect beginning return of function approximately 3 to 7 months after onset of paralysis. If the patient awakens from surgery with a total facial paralysis and then exhibits no evidence of functional return until at least 3 months postsurgery, it is not certain whether axonal degeneration occurred during surgery or in the immediate postoperative period, unless voluntary action potentials are demonstrated on postoperative EMG or unless intraoperative evoked EMG is performed. If we can demonstrate mixed fibrillation and voluntary potentials on postoperative EMG, we can assume direct trauma to some of the axonal fibers yet be assured that total nerve degeneration has not occurred up to that point.

If indeed a progressive nerve degeneration can and does occur with edematous changes in both traumatic and idiopathic palsies, it is not unreasonable to assume it might also occur following surgery for acoustic tumor. In reviewing the literature, there is a scarcity of information concerning immediate compared with delayed paralysis, total nerve degeneration compared with partial nerve degeneration, or recovery times for initial return of facial motion. The explanation for this seems apparent. First, the surgeon does not wish to subject the recouperating patient to noxious examinations such as needle EMG or evoked potential testing. Second, patients are often discharged from referral centers to be followed by their primary physicians in the early postoperative period only to return after extended periods. This is 
reasonable considering the distances that some patients travel. The primary physicians may be inexperienced in observation or testing of nerve recovery. Third, because there is little difference in therapy between the early and the delayed recovery pattern, interest is minimal once the course has already been decided.

Ultimately, surgeons do wish to know if some secondary rehabilitative measure is necessary in patients who do not recover facial function within the expected time frame. We might expect improved long-term facial expression if we could prevent a total nerve degeneration with its usual generalized random misdirection regeneration effects. This rationale stimulated us to consider the prophylactic effects of decompressing the labyrinthine segment of the facial nerve during translabyrinthine acoustic tumor removal. In this study, we compare the rates of postopertive delayed facial weakness in two groups of patients to ascertain whether meatal decompression may be of benefit to patients undergoing acoustic neuroma surgery.

\section{CLINICAL MATERIAL AND METHODS}

Twenty-five patients who were undergoing translabyrinthine acoustic tumor removal received prophylactic decompression of the labyrinthine segment as well as the middle ear and mastoid segment of the facial nerve during their procedures (Group 1, study group). Attention was paid to whether a complete paralysis occurred and to whether early voluntary motion returned within 1 month or if it was delayed longer than 3 months. Data were gathered from 40 other patients (Group 2, comparison group) undergoing consecutive microsurgical removal of acoustic neuroma at the Johns Hopkins Hospital who did not receive prophylactic decompression of the labyrinthine segment. Surgery in all patients in both groups was performed jointly by a neurootological surgeon and a microneurosurgeon. Patients in both groups had received pre- and postoperative steroids in the form of dexamethasone at a dosage of $4 \mathrm{mg}$ four times daily for 4 days and then tapering over 3 or more days. The technique normally used to clarify the separation between the superior vestibular and facial nerves is to identify the ampulla of the superior semicircular canal, and then the vertical crest is used for delineation of the nerves. The method for decompression of the labyrinthine segment, that portion of the facial nerve between the geniculate ganglion and the internal auditory canal, was performed as previously described.[20]

\section{RESULTS}

Total tumor removal was achieved in 22 of the 25 cases in the Group 1. In one patient with a 5-cm tumor, total removal was achieved in a second-stage operation via a suboccipital craniotomy. Two cases were planned subtotal tumor removal in elderly patients. Total removal was achieved in all but one of the 40 patients in Group 2. In 63 of the cases under review, the nerve was anatomically preserved at surgery as described by the surgeons in the operative notes. In two cases facial-facial nerve reconstructions were performed.

To avoid confusing the results, only facial paralysis with subsequent degeneration was examined. Progression to a total facial paralysis from an immediate normal facial expression or slight paresis occurred up to 2 weeks after surgery; however, in most cases progression was immediate or within 5 days. No patient in either group progressed to a total nerve degeneration with delayed recovery if total paralysis began after 8 days. In Group 1, five patients had postoperative paralysis that had not been previously present. Of these five new cases of palsy, three were immediate and two were delayed. Neither of the two cases of delayed palsy progressed to a delayed total nerve degeneration; that is, both had recovery of early voluntary facial motion within 4 weeks of onset. In Group 2 patients, delayed 
degeneration with recovery beginning 3 months following initial good facial function occurred in eight (20\%) of 40 cases. Four of these procedures were performed via suboccipital craniotomy and one via translabyrinthine route. It should be noted that two of the eight tumors in these patients were less than 1.5 $\mathrm{cm}$ in diameter. In summary, none of the patients in Group 1 experienced palsy that progressed to degeneration with delayed recovery longer than 4 weeks, whereas $20 \%$ of Group 2 patients did.

\section{DISCUSSION}

To avoid significant additional surgeries or anesthesia time that might contribute to overall morbidity, the study group was chosen from patients who were already selected for the translabyrinthine procedure. In so doing, an inadvertent difference in tumor size was preselected. The average tumor size in patients undergoing decompression of the labyrinthine segment was $1.8 \mathrm{~cm}$ compared with $2.5 \mathrm{~cm}$ in the control group. A higher ratio of patients who did not undergo decompression of the labyrinthine segment had tumors in the 2- to 4-cm category, whereas most of the patients in Group 1 had tumors in the 0- to 2-cm range (Table 1). There were, however, patients in all tumor size categories in both cases.

\begin{tabular}{|c|c|c|}
\hline \multicolumn{3}{|c|}{$\begin{array}{l}\text { TABLE } 1 \\
\text { COMPARISON OF TUMOR-SIZE CATEGORES IN PATIENTS WH } \\
\text { UNDERWENT ACOUSTIC NEUROMA SURGERY WITH AND WITH } \\
\text { OUT DECOMPRESSION OF THE LABVR NTH NE SEGMENT }\end{array}$} \\
\hline \multirow[b]{2}{*}{ Tum or Size $(\mathrm{cm})$} & \multicolumn{2}{|c|}{ No. of Patierts } \\
\hline & Group 1 & Group 2 \\
\hline $\begin{array}{l}0-2 \\
2-4 \\
>4\end{array}$ & $\begin{array}{r}19 \\
4 \\
2\end{array}$ & $\begin{array}{r}11 \\
24 \\
5\end{array}$ \\
\hline
\end{tabular}

Steroids were administered in all patients and should not influence the differences seen between the two groups. We reinstitute higher-dose steroid administration for an additional period if paresis appears progressive once tapering doses are in effect. In those patients in whom this was indicated, we were impressed by the rapid reversal of the paresis and therefore believe that steroids are of benefit in preventing paralysis. However, in three of the patients in the delayed degeneration categories, steroid medication did not prevent eventual degeneration. Because there are reports in the literature that recovery of facial nerve function occurs up to 3 years after acoustic tumor surgery,[18] we can presume that synkinesis from regenerating axons of partially denervated facial nerves might also worsen gradually over a 3-year period. This lessens confidence in long-term results in relation to the current reported synkinetic recovery grades in some of the patients. On the other hand, it is also not known whether any of the patients in Group 2 might have avoided immediate postoperative paralysis had decompression of the labyrinthine segment been performed. We do not have available data from large series concerning delayed degeneration following acoustic tumor removal.

\section{CONCLUSIONS}

Results of this pilot study suggest that prophylactic decompression of the labyrinthine segment of the facial nerve may be of benefit in patients with acoustic neuroma by preventing total nerve degeneration, especially of the delayed type. More information is needed concerning the natural history of postoperative facial paralysis in current-technique acoustic tumor surgery. Grading of facial nerve results may be most significant if reported after a 2- to 3-year follow up. Finally, further study of the 
decompression of the labyrinthine segment of a prospective nature, matching patients for tumor size, medications, and age, appears to be indicated.

\section{Acknowledgments}

Seventeen of the cases in the comparison group were patients of Dr. David Kennedy of the Department of Otolaryngology-Head and Neck Surgery. Most of the cases in both groups were patients jointly shared with Dr. Donlin Long of the Department of Neurosurgery at the Johns Hopkins Hospital. Other neurosurgeons from his department who shared in the surgeries included: Drs. George Allen, John Rybock, Kennedy Murray, Richard North, and Henry Brem. We wish to thank Ms. Joanne Frantz for her gracious assistance in preparing this manuscript.

Click here to view commentary on this article.

\section{References}

1. Adour KK: The bell tolls for decompression? N Engl J Med 292:748-750, 1975

2. Arriaga MA, Luxford WM, Atkins JS Jr, et al: Predicting long-term facial nerve outcome after acoustic neuroma surgery. Otolaryngol Head Neck Surg 108:220-224, 1993

3. Brackmann DE, Barrs DM: Assessing recovery of facial function following acoustic neuroma surgery. Otolaryngol Head Neck Surg 92:88-93, 1984

4. Cerullo LJ, Grutsch JF, Heiferman K, et al: The preservation of hearing and facial nerve function in a consecutive series of unilateral vestibular nerve schwannoma surgical patients (acoustic neuroma). Surg Neurol 39:485-493, 1993

5. Dobozi M: Surgical anatomy of the geniculate ganglion. Acta Otolaryngol 80:116-119, 1975

6. Esslen E: The Acute Facial Palsies. Berlin: Springer-Verlag, 1977

7. Gantz B, Gmur A, Fisch U: Intraoperative evoked electromyography in Bell's palsy. Am J Otolaryngol 3:272-278, 1982

8. Gianoli GJ, Kartush JM: Delayed facial palsy after acoustic neuroma resection: the role of viral reactivation. Am J Otol 17:625-629, 1996

9. Holliday MJ: Labyrinthine facial nerve decompression in acoustic neuroma surgery. Otolaryngol Head Neck Surg 93:48, 1985

10. House JW, Brackmann DE: Facial nerve grading system. Otolaryngol Head Neck Surg 93:146-147, 1985

11. Lalwani AK, Butt FY, Jackler RK, et al: Delayed onset facial nerve dysfunction following acoustic neuroma surgery. Am J Otol 16:758-764, 1995

12. Lalwani AK, Butt FY, Jackler RK, et al: Facial nerve outcome after acoustic neuroma surgery: a study from the era of cranial nerve monitoring. Otolaryngol Head Neck Surg 111:561-570, 1994

13. Megerian CA, McKenna MJ, Ojemann RG: Delayed facial paralysis after acoustic neuroma surgery: 
factors influencing recovery. Am J Otol 17:630-633, 1996

14. Nutik SL: Facial nerve outcome after acoustic neuroma surgery. Surg Neurol 41:28-33, 1994

15. Peiterson E: Spontaneous course of Bell's palsy, in Fisch U (ed): Facial Nerve Surgery. Birmingham: Aesculapus, 1977

16. Samii M, Matthies C: Management of 1000 vestibular schwannomas (acoustic neuromas): the facial nerve--preservation and restitution of function. Neurosurgery 40:684-695, 1997

17. Sampath P, Holliday MJ, Brem H, et al: Facial nerve injury in acoustic neuroma (vestibular schwannoma) surgery: etiology and prevention. J Neurosurg 87:60-66, 1997

18. Sampath P, Rhines LD, Goel R, et al: Cranial nerve preservation in contemporary vestibular schwannoma (acoustic neuroma) surgery. Crit Rev Neurosurg (In press, 1998)

19. Sargent EW, Kartush JM, Graham MD: Meatal facial nerve decompression in acoustic neuroma resection. Am J Otol 16:457-464, 1995

20. Wedekind C, Hildebrandt G, Klug N: A case of delayed loss of facial nerve function after acoustic neuroma surgery. Zentralbl Neurochir 57:163-166, 1996

Manuscript received August 14, 1998.

Accepted in final form August 26, 1998.

Presented at the Otology Scientific Session of the Annual Meeting of the American Academy of Otolaryngology-Head and Neck Surgery, Inc., Atlanta, Georgia, October 23, 1985.

Address reprint requests to: Prakash Sampath, M.D., Meyer 7-113, The Johns Hopkins Hospital, 600 North Wolfe Street, Baltimore, Maryland 21205. 